\title{
Multi-sensory therapy in psychiatric care
}

\section{Sarah Baillon, Erik van Diepen \& Richard Prettyman}

Multi-sensory therapy is an activity which usually takes place in a dedicated room where patients experience a range of unpatterned visual, auditory, olfactory and tactile stimuli (Baker et al, 1997). These rooms are designed to create a feeling of comfort

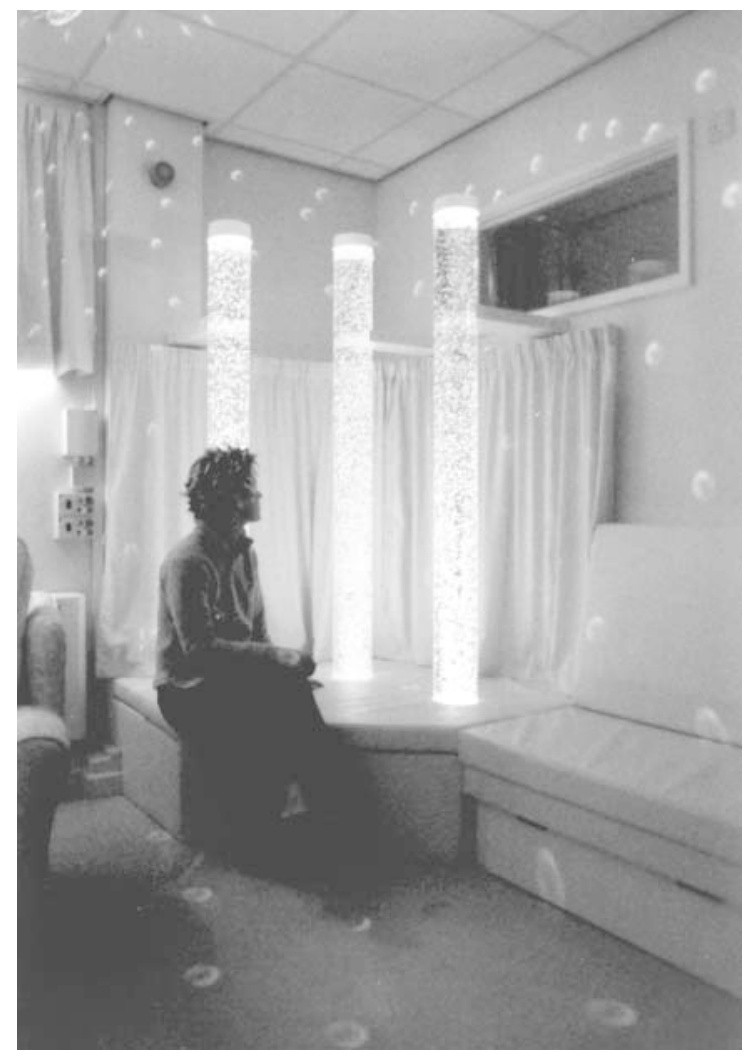

Fig. 1 Bubble-tubes in a multi-sensory environment and safety, where the individual can relax, explore and enjoy the surroundings (Fig 1.).

Multi-sensory environments vary in their appearance and in the equipment they contain, particularly if they are designed to suit the needs of a specific client group. Commonly, the room is painted white or a pastel shade, which optimises the effects of projected light, and it has blacked-out windows that exclude extraneous light. The seating and flooring, in particular, will depend on the type of individuals who will be using the room. Seating may include beanbags or specialised chairs or beds. The floorcovering may be cushioned or textured, or even have lights or pressure-sensitive pads incorporated into it.

Equipment that is common to many multi-sensory environments includes: a mirror ball and coloured spotlights (which project moving coloured shapes around the room); a projector (which projects moving abstract or reminiscent images onto the walls); fibreoptic sprays or curtains (which change colour and can be draped over or held by patients) (Fig. 2); bubble-tubes (a moving stream of bubbles in an illuminated tube of water, which also gives a sensation of vibration when touched); a music system (to play restful or favourite music); an aromatherapy diffuser, panels of interactive knobs and switches that trigger sounds or lights when activated; and a variety of hand-held objects that offer particular tactile or visual sensations to the patient. Staff may also choose to take in samples of food for patients to smell and taste.

Many multi-sensory environments have remotecontrol devices that enable patients to control the equipment themselves. Other examples of multi-

Sarah Baillon is a research associate in the Division of Psychiatry for the Elderly, University of Leicester, based at Leicester General Hospital (Gwendolen Road, Leicester LE5 4PW, UK). Erik van Diepen is a specialist registrar in psychiatry with Leicestershire and Rutland Healthcare NHS Trust, at Leicester General Hospital. Richard Prettyman is a senior lecturer in the Division of Psychiatry for the Elderly, University of Leicester, based at the Bennion Centre, Glenfield General Hospital. He is also a consultant psychiatrist with Leicestershire and Rutland Healthcare NHS Trust. The authors are currently involved in research into the short- and long-term effects of multi-sensory therapy on people with dementia, who have associated agitated behaviour. 


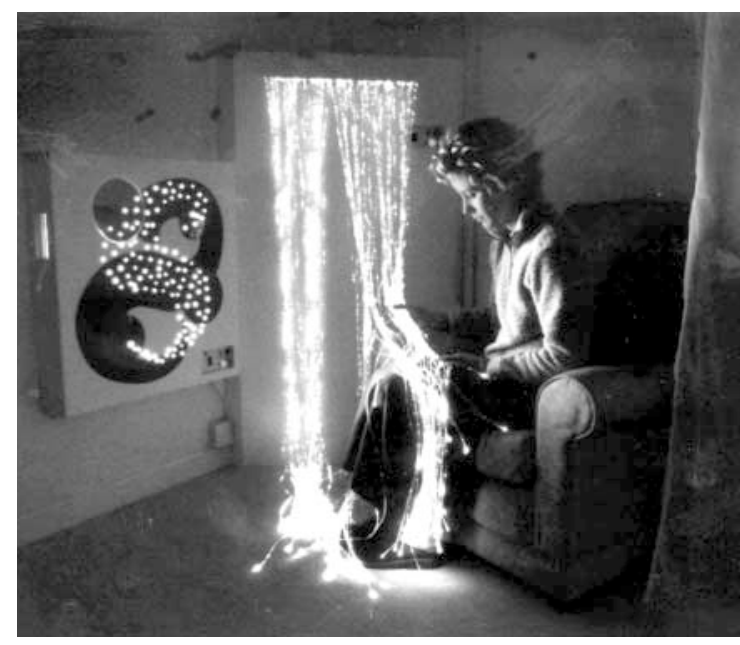

Fig. 2 A fibre-optic spray can be held as it changes colour

sensory environments are soft-play areas, ball pools or specialised spa pools.

In this way, multi-sensory therapy provides stimulation, via the senses of touch, sight, hearing, smell and taste, and also vestibular and proprioceptive stimulation, as the patient moves about the room exploring the equipment (Box 1). Its aim is to be a relaxing activity, designed 'to create a feeling of safety, novelty and stimulation which is under the user's control' (Ashby et al, 1995) and in which there are no expectations for performance (Box 2).

\section{History}

Multi-sensory therapy is a relatively new intervention which is closely related to the ideas behind sensory stimulation therapy. The concept originated in the Netherlands in the 1960s, in the field of learning disabilities, and was first described in the UK by Hulsegge \& Verheul (1987). Early work in this field called this intervention 'snoezelen', which

\section{Box 1 Provisions of multi-sensory therapy}

Stimulation

- visual

- aural

- tactile

- olfactory

- gustatory

- proprioceptive

Control and choice

\section{Box 2 Aspects of multi-sensory therapy}

Relaxation

Stimulation

Failure-free

Non-directive

Responsive to the individual

Enabling

Rapport-building

is a Dutch word derived from an amalgamation of the verbs meaning 'to explore' and 'to relax'. The term 'snoezelen' remains in common use, although the word has now been registered as a trademark by a company which supplies equipment for multisensory environments.

It is difficult to find recreational activities that are enjoyable and age-appropriate for people with learning disabilities. Many conventional therapies from other fields of care are unsuitable for those with severe and multiple physical and mental limitations because they place expectations on the patient to attempt and achieve something that is potentially beyond their abilities. In addition, people with severe and multiple handicaps often experience very limited psychological and sensory stimulation, particularly in institutional care, and have a limited degree of control and choice in all aspects of their lives. Multi-sensory therapy was developed as a leisure resource for people with learning disabilities and designed to be both a relaxing and a stimulating environment which is failure-free (because there is no specific task to be completed, nor goal to achieve) and in which individuals can choose, control and explore the stimuli around them.

Recently, the use of this intervention has extended throughout the world and into other areas of care such as those listed in Box 3. The prevalence of this type of therapy in the UK is hard to estimate as it is becoming increasingly available both within and outside mental health facilities, for example in community facilities and residential homes. In

Box 3 Fields of care in which multi-sensory therapy is becoming widely used

Adult learning disabilities

Dementia care

Children with special needs

Paediatrics

Maternity

Management of chronic pain

Adult psychiatry

Stroke and traumatic brain injury 
addition, many places have portable versions of equipment that is usually found in a dedicated multi-sensory unit. These portable items can be used anywhere (e.g. at the bedside) and can thus incorporate aspects of the philosophy of multi-sensory therapy into the entire care environment.

Some empirical research has been published in learning disabilities, dementia care and chronic pain, but to date (to our knowledge) no empirical studies have been published in other fields of care. The remainder of this article therefore concentrates on those areas for which published evidence exists.

\section{Reported benefits}

\section{Learning disabilities}

Originally, multi-sensory therapy was considered as a useful leisure and recreation facility for patients with learning disabilities but, increasingly, claims are being made regarding the therapeutic benefits of this intervention both in learning disabilities and in other health care fields. However, valid empirical research in this area is limited and it is plagued with methodological problems such as the absence of a control condition, small numbers of subjects, heterogeneous samples and difficulty in measuring relevant outcomes.

Initial research carried out in the learning disabilities area suggests that there may be many ways in which people might benefit from multisensory therapy (Box 4). Studies have varied in the measures that they have used to assess the effects of multi-sensory therapy sessions, but the benefits which have been reported include: positive changes in behaviour (Hutchinson \& Haggar, 1991; Long \& Haig, 1992); improved task concentration (Ashby et al, 1995; Lindsay et al, 1997); an increase in a variety of skills such as awareness of self, social interaction behaviours, communication, exploration and

\section{Box 4 Reported benefits of multi-sensory therapy in learning disabilities}

Improved task concentration

Improved awareness of self

Greater social interaction and communication

Increase in exploration and manipulation of stimuli

Increase in adaptive behaviours

Reduction in stereotypic self-stimulatory behaviours

Improved staff morale manipulation of stimuli (Houghton et al, 1998); relaxation (Slevin \& McClelland, 1999); and a reduction in stereotypic self-stimulatory behaviours and an increase in adaptive behaviours such as exploratory behaviour or initiating contact with others (Shapiro et al, 1997).

However, only three of the above studies included a control condition. Two of these (Lindsay et al, 1997; Shapiro et al, 1997) reported positive benefits (reduction of stereotypic self-stimulatory behaviour/ increased concentration) of multi-sensory therapy sessions over the control intervention, but one (Martin et al, 1998) concluded that they could not confirm that multi-sensory therapy had 'any effects beyond those that could be ascribed to the social interaction between the participant and the enabler'. However, this research did rely on measures which took place a week after the end of the interventions and so may have failed to demonstrate short-term changes in behaviour.

In addition to the possible benefits to the mood and behaviour of patients, many authors have also reported that multi-sensory therapy promotes a close therapeutic relationship and rapport between the patient and the carer or staff member who participates in the sessions. It is often beneficial for the carer to share the experience of a non-dependent, non-caregiving activity with the patient. Spending time in the multi-sensory therapy environment with a patient can help the carer or staff member to interpret how the patient is feeling and what his or her preferences are - this knowledge can assist their care of that patient outside the multi-sensory environment. It has also been suggested that the experience of doing something positive and spending 'quality time' with a patient serves to raise staff morale and reduce staff burn-out (Morrissey \& Biela, 1997). Multi-sensory therapy provides an opportunity for carers to focus on the preferences of patients and legitimises spending time with them, and this has a positive effect on their relationship with their patients.

More research is needed to establish and evaluate the benefits that multi-sensory therapy may have to offer people with learning disabilities. Existing research is promising and tends to support the strong feelings and anecdotal evidence from care professionals in the field, who describe the positive results they have seen. However, valid empirical evidence is lacking, and although provision of multisensory therapy facilities is fast becoming standard in learning disability care units throughout the UK, there remains a need to establish a body of rigorous research to help us understand its effects and justify its widespread use (Mount \& Cavet, 1995). The concept of multi-sensory therapy does have its opponents, who argue that it serves to perpetuate 
the segregation of people with learning disabilities from normal experiences and sensations, and that keeping them in specialist units and environments goes against the principles of 'normalisation' (Whittaker, 1992). Conversely, others argue that multisensory therapy is in keeping with the concept of normalisation, in that it provides access to an appropriate leisure activity for those with disabilities and is an activity that can be enjoyed equally by people with or without disabilities (Cunningham et al, 1991; Haggar \& Hutchinson, 1991).

\section{Dementia care}

Various published research and anecdotal reports have suggested that multi-sensory therapy can have a positive effect on patients with dementia and related behavioural problems (Box 5). However, as in the field of learning disabilities, valid empirical research in this area is limited owing to studies that lack control conditions and/or have small numbers of subjects. This research has indicated that multisensory therapy may have a positive effect on the mood of those with dementia, in terms of an increase in observers' ratings of happiness, enjoyment and relaxation, and a reduction in sadness, fear and boredom (e.g., Moffat et al, 1993; Pinkney, 1997; Baker et al, 1998). These and other studies have also indicated that multi-sensory therapy may increase patients' attentiveness to their environment (Moffat et al, 1993; Baker et al, 1998; Spaull et al, 1998), increase appropriate communication (Baker et al, 1998) and reduce the occurrence of socially disturbed and challenging behaviour (Kragt et al, 1997; Spaull et al, 1998). However, out of all the above studies, only two (Pinkney, 1997; Baker et al, 1998) included an appropriate control condition and only one (Baker et al, 1998) included a suitable number of subjects. Baker et al concluded that multi-sensory therapy is particularly appropriate for people with moderate or severe dementia, where more structured approaches may fail, and they reported that the staff involved felt that it promoted relaxation in agitated patients and stimulated unresponsive ones.

Box 5 Reported benefits of multi-sensory therapy in dementia care

Increased happiness, enjoyment and relaxation Reduction in sadness and fear Increased attentiveness to environment Increased appropriate communication Reduced disturbed behaviour Improved staff morale
As in learning disabilities, many of the above studies refer to the positive effect that multi-sensory therapy can have on staff morale and relationships with patients by providing the opportunity to share a non-instrumental activity and gain insight into the individuals for whom they care.

\section{Pain management}

There is much published research into the use of multi-sensory therapy in the management of chronic pain (e.g. Schofield, 2000), and it has been shown to reduce recorded pain levels, depression and disability of functioning (e.g. physical, psychosocial, sleep) and to help sufferers cope.

\section{Possible drawbacks}

It is apparent that not all individuals like or benefit from multi-sensory therapy and also that an individual's response can vary on different occasions. For some, the stimulating environment may have the effect of increasing the existing level of agitation; for others, or for the same individual on a different occasion, it may have a calming effect. Some patients find the unusual visual effects confusing and can become distressed. The skill of the therapist in introducing the patient to the multisensory environment in the correct manner appears to be an important factor in the patient's response to multi-sensory therapy, but it by no means guarantees a positive response. The therapist must be sensitive to the patient's reaction to the environment on each occasion and take the appropriate measures to ensure that the person does not become distressed.

\section{How does multi-sensory therapy work?}

Various theories have been put forward to account for how and why multi-sensory therapy can have positive effects for people with multiple handicaps.

\section{Sensory deprivation/stimulation}

Research carried out in the 1960s showed that prolonged or frequent restriction of appropriate and understandable stimulation can lead to negative psychological outcomes for individuals (Zuckerman, 1964). It is not only the amount of stimulation that 
is important, but also variation in the stimulation, i.e. monotonous unchanging stimulation can be as negative as no stimulation at all. Many care settings in which people with dementia or learning disabilities spend much of their time can be unstimulating or offer no variation in stimulation. Physical, sensory and cognitive impairments further reduce the amount of meaningful stimulation that an individual receives. Impaired cognitive ability also restricts an individual's ability to make sense of the stimulation that is received. Such deprivation of meaningful sensations can lead to negative outcomes such as anxiety, stress, depression, withdrawal and reduced motivation, or agitation and disturbed behaviour. Specific stimulation of the primary senses in an environment that excludes all extraneous stimulation makes perception and interpretation of those sensations easier for patients and alleviates the effects of this deprivation. The stimulation can then be adapted according to the individual's responses to it, thus making the experience increasingly appropriate and positive.

\section{Demand-free environment}

Interpretation of a complex environment makes demands on an individual with sensory and cognitive impairments, as do many recreational activities. Baker et al (1998) propose that multi-sensory therapy requires no memory or cognitive reasoning ability. This removes demands on individuals to understand what they are experiencing, thus reducing the tendency for them to feel confused and to withdraw. In addition, the stimuli presented in multi-sensory therapy are unpatterned and thus less demanding of attention and cognitive processing. This therefore reduces the demands and stress that individuals are under and encourages more positive and appropriate behaviour.

\section{Learned helplessness}

Individuals with severe and multiple disabilities often have little or limited opportunity to exert control over the environment around them. As a result of this experience they can feel helpless, lose confidence that they are able to exert any influence on their environment (even when they are able to take some level of control) and become withdrawn and apathetic. In effect, they learn to be more helpless than their disabilities actually make them.

The multi-sensory therapy environment is safe and failure-free. Individuals are given the opportunity to exert and experience control over their environment. This may be through direct interaction with the equipment, through use of a specially adapted remote control or by communicating their preferences to an accompanying staff member. Through this experience, patients are given the feeling of independence and choice. Many clinicians feel that this aspect of multi-sensory therapy is an important factor in how the intervention works.

\section{Social contact}

There is a debate as to how much the intervention itself brings about the positive changes in patients and how much they are attributable to the change that multi-sensory therapy makes to the relationships between staff and patients (Mount \& Cavet, 1995). In hospital and other care environments, elderly patients are often involved in very little staffpatient interaction, particularly in terms of social activities and prolonged informal conversations (Armstrong-Esther et al, 1994). Multi-sensory therapy may legitimise informal, non-instrumental contact between staff and patients, encouraging and enabling staff to increase the amount of time they spend interacting with their patients (Ellis \& Thorn, 2000). The philosophy of multi-sensory therapy also makes staff reconsider and be aware of ways to communicate with patients as individuals, and this engenders feelings of well-being in both patients and staff (Hope, 1996). Multi-sensory therapy may therefore influence how staff spend time with patients, as well as their awareness of ways to communicate and their relationship with them.

Whatever mechanism makes multi-sensory therapy helpful, it is agreed that it is essential for all individuals to have access to appropriate pleasurable experiences which are under their own control, and that this is fundamental to a person's wellbeing. Multi-sensory therapy enables carers to cater for this need in a way that is appropriate for individuals who have cognitive, physical and perceptual impairments. The enabling, non-directive, non-goaloriented aspects of multi-sensory therapy are also important to encourage individuals' engagement with and awareness of their surroundings and to promote motivation and feelings of control and choice, which may be limited in many other aspects of their lives.

\section{Practical considerations}

A large number of articles in the occupational therapy and nursing literature refer to the importance of many practical issues relating to the use of multi-sensory therapy with patients who have learning disabilities or dementia. For example, there 
are problems surrounding the issue of gaining consent to participate from such patients, when it is difficult to explain what the multi-sensory environment is like. The use of photographs can be helpful in explaining the concept to them and gentle introduction to the room (e.g. not having all the equipment turned on at once), combined with sensitive observation of their reactions, can ensure that patients are willing to participate and are open to the experience.

It is imperative that the staff who accompany patients in a multi-sensory environment are familiar with them, their background, health and perceptual problems, and the ways in which they express their discomfort and preferences. There may be issues for patients regarding their cultural background, their openness to therapeutic and non-therapeutic touch (invasion of personal space) and the gender of the staff. With this knowledge, staff can arrange the session in a way that is appropriate for the individual, sensitive to his or her boundaries and responsive to preferences.

Planning and equipping a multi-sensory environment must be undertaken with careful consideration of the needs of the patient group for whom it is designed. Consideration also needs to be given to health and safety issues and to training staff in the use of the equipment and in facilitating sessions. Problems which can affect the use of multi-sensory environments in some units include: limited staff availability to accompany patients to the room; the location of the room in relation to the care environment (patients may be distressed by having to leave their usual care environment or by the distance that they have to travel); and staff being unclear about which patients are most likely to benefit.

\section{Summary}

Given the ever-increasing popularity of this new activity, it is important that further systematic research is undertaken to provide evidence regarding the efficacy of multi-sensory therapy in all of the fields of care in which it is currently being used. It remains to be established whether conventional relaxation techniques or other approaches (such as enhancing the sensory experiences of the everyday environment) could be as effective in achieving the same benefits. The reported benefit that multisensory therapy may be useful in reducing behavioural problems has important implications in view of the limited efficacy of pharmacological treatment for these symptoms (Schneider, 1996), which have been shown (e.g. Kaufer et al, 1998; Haupt \& Kurz, 1993) to cause the most distress in carers of those with dementia and contribute greatly to the decision to place patients in long-term residential care.

Some people feel that the current lack of empirical evidence fails to justify the widespread use and expense of multi-sensory facilities and there is a clear need to prove that patients do benefit from multisensory therapy and are not just passive recipients of the intervention (Woodrow, 1998). It is perhaps worth noting, however, that many interventions (such as reminiscence, reality orientation and validation therapy) which are long-established and widely used in dementia care also lack a background of rigorous research evidence.

The research literature into the relative merits of multi-sensory therapy may not be substantial, but it does indicate support for the large amount of anecdotal evidence which claims that this activity is an effective and appropriate therapeutic intervention for people in mental health care.

\section{References}

Armstrong-Esther, C. A., Browne, K. D. \& McAfee, J. G. (1994) Elderly patients: still clean and sitting quietly. Journal of Advanced Nursing, 19, 264-271.

Ashby, M., Lindsay, W. R., Pitcaithly, D., et al (1995) Snoezelen: its effects on concentration and responsiveness in people with profound multiple handicaps. British Journal of Occupational Therapy, 58, 303-307.

Baker, R., Dowling, Z., Wareing, L. A., et al (1997) Snoezelen: its long-term and short-term effects on older people with dementia. British Journal of Occupational Therapy, 60, 213218

-, Bell, S., Assey, J., et al (1998) A Randomised Controlled Trial of the Snoezelen Multi-Sensory Environment for Patients with Dementia. Bournemouth: Dorset HealthCare NHS Trust.

Cunningham, C. C., Hutchinson, R. \& Kewin, J. (1991) Recreation for people with profound and severe learning difficulties: the Whittington Hall Snoezelen Project. In The Whittington Hall Snoezelen Project. A Report from Inception to the End of the First Twelve Months (ed. R. Hutchinson). Chesterfield: North Derbyshire Health Authority.

Ellis, J. \& Thorn, T. (2000) Sensory stimulation: where do we go from here? Journal of Dementia Care, 8, 33-37.

Haggar, L. E. \& Hutchinson, R. B. (1991) Snoezelen: an approach to the provision of a leisure resource for people with profound and multiple handicaps. Mental Handicap, 19, 51-55.

Haupt, M. \& Kurz, A. (1993) Predictors of nursing home placement in patients with Alzheimer's disease. International Journal of Geriatric Psychiatry, 8, 741-746.

Hope, K. (1996) Caring for older people with dementia: is there a case for the use of multisensory environments? Reviews in Clinical Gerontology, 6, 169-175.

Houghton, S., Douglas, G., Brigg, J., et al (1998) An empirical evaluation of an interactive multi-sensory environment for children with disability. Journal of Intellectual and Developmental Disability, 23, 267-278.

Hulsegge, J. \& Verheul, A. (1987) Snoezelen Another World. Chesterfield: ROMPA.

Hutchinson, R. \& Haggar, L. (1991) The development and evaluation of a Snoezelen leisure resource for people with profound and multiple handicaps. In The Whittington Hall Snoezelen Project. A Report from Inception to the End of the First Twelve Months (ed. R. Hutchinson). Chesterfield: North Derbyshire Health Authority. 
Kragt, K., Holtkamp, C. C. M., Van Dongen, M. C. J. M., et al (1997) Het effect van snoezelen in de snoezelruimte op het welbevinden van demente ouderen. Verpleegkunde, 12, 227236.

Kaufer, D. I., Cummings, J. L., Christine, D., et al (1998) The impact of neuropsychiatric syptoms in Alzheimer's disease: the Neuropsychiatric Inventory Caregiver Distress Scale. Journal of the American Geriatrics Society, 46, 210-216.

Lindsay, W. R., Pitcaithly, D., Geelan, N., et al (1997) A comparison of the effects of four therapy procedures on concentration and responsiveness in people with profound learning disabilities. Journal of Intellectual Disability Research, 41, 201-207.

Long, A. P. \& Haig, L. (1992) How do clients benefit from Snoezelen? An exploratory study. British Journal of Occupational Therapy, 55, 103-106.

Martin, N. T., Gaffan, E. A. \& Williams, T. (1998) Behavioural effects of long-term multi-sensory stimulation. British Journal of Clinical Psychology, 37, 69-82.

Moffat, N., Barker, P., Pinkney, L., et al (1993) Snoezelen. An Experience for People with Dementia. Bournemouth: Dorset Health Care NHS Trust.

Morrissey, M. \& Biela, C. (1997) Snoezelen. Benefits for nursing older clients. Nursing Standard, 12, 38-40.

Mount, H. \& Cavet, J. (1995) Multi-sensory environments: an exploration of their potential for young people with profound and multiple learning difficulties. British Journal of Special Education, 22, 52-55.

Pinkney, L. (1997) A comparison of the Snoezelen environment and a music relaxation group on the mood and behaviour of patients with senile dementia. British Journal of Occupational Therapy, 60, 209-212.

Schneider, L. S. (1996) Meta-analysis of controlled pharmacologic trials. International Psychogeriatrics, 8 (suppl. 3), 375379.

Schofield, P. (2000) The effects of Snoezelen on chronic pain. Nursing Standard, 15, 33-34.

Shapiro, M., Parush, S., Green, M., et al (1997) The efficacy of the 'snoezelen' in the management of children with mental retardation who exhibit maladaptive behaviours. The British Journal of Developmental Disabilities, 43, 140-155.

Slevin, E. \& McClelland, A. (1999) Multisensory environments: are they therapeutic? A single-subject evaluation of the clinical effectiveness of a multisensory environment. Journal of Clinical Nursing, 8, 48-56.

Spaull, D., Leach, C. \& Frampton, I. (1998) An evaluation of the effects of sensory stimulation with people who have dementia. Behavioural and Cognitive Psychotherapy, 26, 77-86.

Whittaker, J. (1992) Can anyone help me to understand the logic of Snoezelen? Community Living, October, p. 15.

Woodrow, P. (1998) Interventions for confusion and dementia. 4. Alternative approaches. British Journal of Nursing, 7, 12471250

Zuckerman, M. (1964) Perceptual isolation as a stress situation. Archives of General Psychiatry, 11, 255-276.

\section{Multiple choice questions}

1. Multi-sensory environments:

a are strictly standardised to suit a particular client group

b provide visual, olfactory and proprioceptive stimuli

c aim to encourage patients to explore

d aim to create a relaxing atmosphere

e always place the equipment under the control of the therapist.

2. In the research carried out in the learning disabilities field, multi-sensory therapy has been reported to have a positive effect on:
a task concentration
b communication
c apraxia
d self-stimulatory behaviour
e relaxation.

3. The therapeutic benefits of multi-sensory therapy are possibly related to:

a being task-oriented and so encouraging a higher level of functioning

b the patient having a sense of being in control of their environment

c giving stimulation to all the senses simultaneously

$\mathrm{d}$ the stimuli being unpatterned in nature

e exclusion of extraneous stimuli.

4. Research carried out in dementia care indicates that multi-sensory therapy can improve:
a mood
b orientation in time
c relaxation
d staff morale
e mobility.

5. In multi-sensory therapy:

a knowledge of the patient is important

b it is not necessary to gain a patient's consent

c anyone can accompany a patient in the multisensory environment - no training is required

$\mathrm{d}$ the patient must be able to communicate verbally

e all patients will benefit.

\section{MCO answers}

1

a $\mathbf{F}$

b $\mathbf{T}$

c $\mathbf{T}$

d $\mathbf{T}$

e $\mathbf{F}$
2

a $\mathbf{T}$

b $\mathbf{T}$

c $\mathbf{F}$

d $\mathrm{T}$

e $\mathbf{T}$

\section{3}

a $\mathbf{F}$

b $\mathrm{T}$

c $\mathrm{F}$

d $\mathbf{T}$

e $\mathbf{T}$
4

a $\mathrm{T}$

b $\mathrm{F}$

c $\mathrm{T}$

d $T$

e $\mathbf{F}$
5

a $\mathbf{T}$

b $\mathbf{F}$

c $\mathrm{F}$

d F

e $F$ 